\title{
Induction of anti-apoptotic signaling via ATF4 confers resistance to the inhibition of essential amino acid transporter LAT1
}

\author{
Takashi Yamaga, Junichi Suehiro, Hiroyuki Sakurai \\ Department of Pharmacology, Kyorin University School of Medicine, Japan
}

Neutral amino acid transporter LAT1 overexpressed in many cancer cells is an attractive therapeutic target because it provides cancer cells with essential amino acids required for proliferation. JPH203, a specific competitive inhibitor for LAT1, has been reported to suppress proliferation of cancer cells including pancreas, colon, head and neck, and prostate. Recently, we found that JPH203 robustly inhibited essential amino acid uptake of several breast cancer cell lines including MDA-MB-231 cells, a model of high-grade malignant triple-negative breast cancer cells (TNBC) and 3 non-TNBC cell lines. Despite marked reduction of amino acid uptake, JPH203 at $100 \mu \mathrm{M}$ only slightly suppressed MDA-MB-231 cell viability assessed by MTT assay to 60\% of vehicle treated cells. In contrast, the same concentration of JPH203 efficiently suppressed non-TNBC T-47D cell line to $20 \%$ of vehicle treated cells. Flow cytometry-based apoptosis detection assay showed that JPH203 at $100 \mu \mathrm{M}$ did not trigger externalization of annexin- $\mathrm{V}$ in MDA-MB-231 while it increased annexin$\mathrm{V}$-positive fraction to $60 \%$ in T-47D cells. We hypothesized that this observed difference in antitumor effects of JPH203 between MDA-MB-231 cells and T-47D cells may be due to different transcriptional response to nutrient stress (i.e. essential amino acid starvation) between them. We thus analyzed global gene expression change in JPH203-responsive T -47D cells in the presence or absence of JPH203 using DNA microarray. Differentially expressed genes (more than 2 fold increase/decrease) belong to amino acid metabolism, cell proliferation, amino acid synthesis and stress response ontologies. Among them, we focused on ATF4, a master transcription factor for stress response, which activates antiapoptotic pathway. Knockdown of ATF4 in MDA-MB-231 cells, but not in T-47D cells, enhanced JPH203 induced cell death. These results suggest that nutrient stress caused by JPH203-treatment activated anti-apoptotic signaling via ATF4 in MDA-MB-231 but not in T-47D cells, and that ATF4 inhibition could be a novel way of breaking resistance to antiLAT1 therapy in MDA-MB-231 cells. 